\title{
Erratum to: Congenital HCMV and assisted reproduction: Why not use the chance for primary screening?
}

\author{
Christiane Kling ${ }^{1} \cdot$ Dieter Kabelitz $^{1}$
}

Published online: 5 October 2015

(C) Springer-Verlag Berlin Heidelberg 2015

\section{Erratum to: Arch Gynecol Obstet (2015) 291:1205-1211 DOI 10.1007/s00404-014-3583-z}

In our recently published manuscript we proposed a diagnostic workflow on primary prevention of HCMV infection in a special group of prospective parents who conceive under infertility treatment and live in a low HCMV prevalence setting. In this group it is possible to prevent primary infection of the mother and congenital HCMV disease. Detection is based on the relatively cheap and unambiguous test for HCMV-IgG antibodies. The schedule comprises three steps: At the initial assessment, results of serological testing and a questionnaire lead to an estimation of the annual seroconversion rate (ASR) of the seronegative woman or of the risk for congenital HCMV infection (CHI) in seropositive mothers, as derived from the literature. In our opinion, seronegative women living with a seropositive partner are not regarded to be at high risk and do not require special care (Fig. 1, see *). Women at "high" risk (ASR exceeding $1 \%$ ) are selected for repeated screening before each treatment cycle. In case of seroconversion, they are re-assessed, and the cycle is postponed. Thirdly, those "high risk" women who do not seroconvert but maintain their basal risk for primary infection are screened in the first half of pregnancy. If

The online version of the original article can be found under doi:10.1007/s00404-014-3583-z.

Christiane Kling

christiane.kling@uksh.de

1 Institute of Immunology, University Hospital Schleswig-

Holstein Campus Kiel, Arnold-Heller-Str 3 Haus 17,

24105 Kiel, Germany primary maternal HCMV infection is proven or CHI suspected, mother and fetus should be evaluated according to national guidelines in a specialised centre (Fig. 1).

As we have argued, the chance to detect a primary HCMV infection in either parent is expected to be very low in an area with low HCMV prevalence. Therefore we regarded the time span between initial assessment and first treatment cycle to be long enough for reaching the latent phase should a primary infection have been present at the initial test.

In order to reach a high standard of security as demanded in fertility medicine, this may not be sufficient. Therefore, a second test for avidity of HCMV-IgG antibodies is implemented into the schedule. Whether or not it should be performed in our opinion depends on the results of the questionnaire. A proposal for this questionnaire is attached (Fig. 2). We presume that most seronegative couples undergoing infertility treatment are not at risk for primary infection as long as they do not have children of their own. The main goal of the schedule is to enhance awareness for HCMV, and to provide reassurance. Repeated screening before treatment cycles or in early pregnancy will only rarely be necessary but results will be easier to interpret than those obtained incidentally in pregnancy. Thus precautious elective termination of pregnancy can be prevented. 


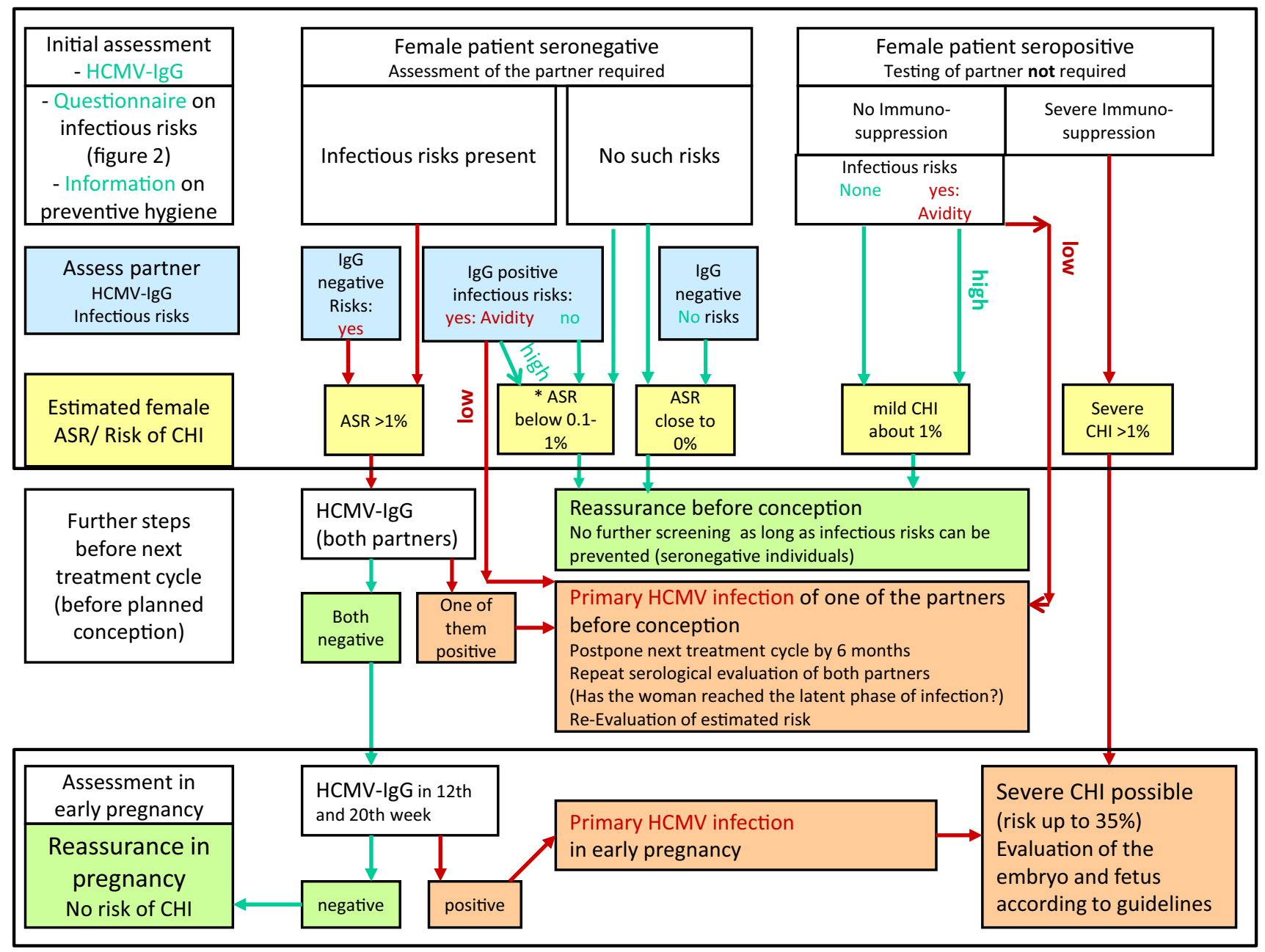

Fig. 1 Diagnostic steps and decisions in HCMV screening

Fig. 2 Possible risks for primary HCMV infection
Possible risks for primary HCMV infection: Proposals for a questionnaire

(1) Personal data (both partners):

- Occupational characteristics (both partners)

- Do you suffer from severe immunosuppression (e.g. HIV, leukemia)?

- Are you under pharmacological immunosuppression (e.g. for autoimmune disease, after transplantation)? Which medication and doses?

- Are you on haemodialysis for chronic renal failure?

(2) Close contact to infants and children below 3 years of age

- Do you have children below 3 years in your household?

- Do they attend a day-care centre?

- Do you yourself care for other children of this age group? (neighbours, family, friends)

- Are you a child-care worker or professional health care worker yourself?

(3) Close contact to people under immunosuppression who may be HCMV positive

- Are there other persons in your household who suffer from immunosuppression (see 1.2-4)?

- Do you care for other persons who suffer from these diseases?

(4) Close contact of the seronegative male partner to infants and children below 3 years of age or to people under immunosuppression

- Do the question under (2) and (3) apply?

Any question answered with "yes" indicates an elevated risk for primary HCMV infection 